\title{
Python app for drawing Bode diagram asymptotes of transfer function for minimum and non-minimal phase systems
}

\author{
Magno Enrique Mendoza Meza ${ }^{1}$ \\ ${ }^{1}$ Federal University of the ABC Engineering Modeling and Applied Social Sciences Center
}

November 23, 2021

\begin{abstract}
The purpose of this article is to introduce an application to draw the asymptotes of Bode diagram module and phase from each constituent elementary factors of any transfer function for minimum and non-minimal phase systems without transport delay. The Bode diagram is the most used tool in the frequency response method. Python was used to program the application to perform the operations as well as the Qt5 Design for the simple graphical interface for the application and all this in the Linux operating system. The application purpose is to assist students in learning the concept and drawing of Bode diagram. For students the non-minimum phase system Bode diagram is more difficult to draw than a minimum phase system due to the presence of zeros and/or poles on right half of s-plane. The phase asymptotes of a quadratic factor was closest to the real phase curve around the corresponding undamped natural frequency and this can be observed in the example showed in this article. This example must be used as a help and not a simply to solve a problem.
\end{abstract}

\section{Hosted file}

wileyNJD-AMA.pdf available at https://authorea.com/users/447549/articles/546520-python-appfor-drawing-bode-diagram-asymptotes-of-transfer-function-for-minimum-and-non-minimalphase-systems 